\title{
NON-VERBAL REASONING IN FIGURATIVE TREATMENT A CORRELATION BETWEEN THE PROCESSES OF RESEARCH AND DRAWING: A CASE STUDY OF SADEQUAIN
}

Umaira Hussain Khan $^{*}$

\begin{abstract}
This paper draws a correlation between processes of research and drawing by analyzing the formation of emotional content and stylistic representation in art. The paper suggests that research process fundamentally involves a systematic development of understanding on a particular issue through a process of rational inquiry. The research outcome or an intellectual understanding is therefore nothing more than a thoroughly investigated form of a hypothesis/ premise/ theory/ idea that has undergone a careful process of scrutiny, comparison and evaluation. On similar grounds, drawing process also involves a systematic development of form in which adjustments are made with the help of nonverbal reasoning till the final form is evolved. The development of form in drawing becomes a systematic rational process but operates at a subconscious plane; reason is substituted by aesthetic sensibility. It is suggested that aesthetic sensibility is a judgment that the human mind tailors through the use of non-verbal criteria of evaluating the beautiful and ugly. The paper develops a theoretical model in the light of above and then applies it to analyze various drawing conventions used by Sadequain in figurative treatment.
\end{abstract}

Keywords: Aesthetic sensibility, drawing conventions, emotional processes, evolutionary, hierarchy, metaphor, neurobiologists, non-verbal reasoning, rationality, subconscious

\section{Introduction}

Drawing and research refer to two different processes that entail two different set of skills but this is to see, that the underlying psychological processes, governing the usage of acquired skills to a specific end, are quite similar and occupy the same domain, i.e. the human mind. Before identifying the psychological processes and to see, how they govern skills of drawing and research, it is necessary to come up with a working definition of how the human mind works particularly when it is engaged in a creative process and is inclined to produce a product. It is also necessary to see the Non-Verbal Reasoning and its Role in Art.

\section{Literature Review}

The correlation between the processes of research and drawing is increasingly becoming explicable in the light of new knowledge that we are gaining about the workings of human mind. In this context various new scientific advances are significant. For instance, the work by neurobiologists such as Antonio Damasio is quite relevant in understanding

\footnotetext{
* Umaira Hussain Khan, Ph.D, Assistant Professor Fine Arts, Department of Visual Studies, Faculty of Arts and Social Sciences, University of Karachi, Karachi
} 
the function of various departments in the human mind. In Looking for Spinoza, Damasio explains the hierarchy of mental functions through a metaphor he calls 'the Nesting Principle'. The metaphor is quite useful and explains how in the course of evolution various mental processes preceded others and in due course an architecture of mental processes evolved. This insight is of particular relevance since it focuses on those mental functions which play key roles in the processes of research and drawing. Another important insight put forth by Bruce Lipton in Biology of Belief has been the difference between information processing capability of the verbal and non-verbal processes of the mind. It has been suggested that emotional processes are much more efficient data processing functions and are important in understanding the functioning of mind. This insight, as we shall see, also holds great relevance for finding common ground between the processes of research and drawing. ${ }^{1}$

The following research also revolves around the question that what kind of rationality is deployed in research and drawing. In respect of this question, Rationality and Emotions in Decision Making by Olga Markic is a significant study. The study informs about the nature of rationality and its various types and also suggests the role that emotions play in influencing a rational choice. This particular area has also been highlighted in a research paper entitled Evolutionary Psychology and the Emotions. The scientific data clearly directs our attention to the administrative hierarchy of the human mind and since the mind controls and determines the quality of drawing and research, therefore a consideration of these insights clarifies the processes in a more understandable way. It is through various interdisciplinary approaches to the human mind that a correlation between various practices of human beings can now be seen taking place in a common ground. The whole of the mind comes into play but with each practice, the set of skills and conventions is differentiated but the overall mechanics display an underlying integration and commonality. The following discussion expands on this very idea and seeks to establish a correlation between research and drawing as two mental processes trading on similar grounds.

\section{A Working Definition of Human Mind}

The human mind is probably the most complex system in the known Universe. According to a generally agreed definition, the human mind can be seen as a data processing system. From the perspective of Evolutionary Psychology it can be defined as a set of information-processing procedures that are physically embodied in the neural circuitry of the brain. ${ }^{2}$ According to evolutionary scientists, 'An evolutionary perspective leads one to view the mind as a crowded zoo of evolved, domain-specific programs. Each is functionally specialized for solving a different adaptive problem that arose during hominid evolutionary history'. ${ }^{3}$ There is a horde of technical detail about its functions coming from a variety of disciplines such as evolutionary biology, neuroscience, psychology, computer sciences, quantum physics and microbiology. For present

\footnotetext{
${ }^{1}$ Lipton, Bruce. The Power of the Mind. January 1, 2008. http://www.newdawnmagazine.com/articles/thepower-of-the-mind (accessed October 15, 2014).

${ }^{2}$ Tooby, Leda Cosmides \& John. Evolutionary Psychology and the Emotions. January 13, 2000, 2.

http://www.cep.ucsb.edu/primer.html (accessed October 8, 2014)

${ }^{3}$ Ibid.
} 
purposes, focus is not into an exhaustive account of what happens in the mind when it is creatively engaged but restricts ourselves to define two aspects. One is a broad division of mental processes based on a general classification of mind and the other is identification of mental processes that play a governing role in the process of research and drawing.

\title{
Non-Verbal Reasoning and its Role in Art
}

Modern scientific literature suggests the human mind, after the emergence of language and civilization, can be seen as a composite of verbal and non-verbal mental processes. Non-verbal processes (emotional processes) are much more efficient and powerful than verbal processes (intellectual processes). The reason is that non-verbal processes come first in the evolutionary ladder and occupy more than ninety percent of the mental functioning. This research shows that only a small portion of our mental activity (such as gathering information, learning \& thinking) is conscious; the majority of our mental activity is entirely non-conscious. These results fully support a statement made about 20 years ago in an influential Cognitive Psychology textbook: "Most of what we do, goes on unconsciously. It is the exception, not the rule, when thinking is conscious, but by its very nature [i.e., because we cannot experience anything else], conscious thought seems the only sort. It is not the only sort; it is the minority".

The verbal processes developed as an extension to aid non-verbal processes by providing information acquired through the capability of verbal abstraction or verbal language. Verbal processes appear to be independent since our consciousness about the working of mind is limited. We cannot possibly make the non-verbal processes tangible to our consciousness since the complexity involved far exceeds our verbal capability of computation. The verbal and non-verbal domains of mental functioning are also referred to as subconscious (non-verbal) and conscious (verbal) divisions of mind. To get an idea of scale, the following quote from an article by Bruce Lipton serves the purpose:

\begin{abstract}
The power of the subconscious mind lies in its ability to process massive amounts of data acquired from direct and indirect learning experiences at extraordinarily high rates of speed. It has been estimated that the disproportionately larger brain mass providing the subconscious mind's function has the ability to interpret and respond to over 40 million nerve impulses per second. In contrast, it is estimated that the diminutive selfconscious mind's prefrontal cortex can only process about 40 nerve impulses per second. As an information processor, the subconscious mind is one million times more powerful than the self-conscious mind. ${ }^{5}$
\end{abstract}

The point to note is that the apparent decision making in the verbal domain involves a multiplicity of governing processes that operate at the non-verbal level. This fact has been endorsed by contemporary Neuroscience. According to Antonio Damasio, our rational decisions are influenced by the same mental processes that give rise to emotions

\footnotetext{
${ }^{4}$ Lewicki, Paul. Conclusions of the Research on Nonconscious Information Processing. n.d. 3 July 2013. ${ }^{5}$ Lipton, Bruce. The Power of the Mind. January 1, 2008, 1. http://www.newdawnmagazine.com/articles/thepower-of-the-mind (accessed October 15, 2014).
} 
and feelings. He has suggested a model that suggests that our rational conclusions and logical judgments are colored by emotions without being aware of it. ${ }^{6}$ It may be proposed that emotional judgments and rational judgments are not modes that are wide apart but processes that are integrally interconnected. And since emotions are more efficient calculators; therefore, the rational processes are most of the times governed by them. This scenario informs that creative engagement of mind, to produce a verbal or aesthetic product, involves processes that have an underlying unity. In the outer world, it may distinguish between a research paper and a drawing but in the internal world of mind the processes that go into the making of these products borrow from same sources.

In the process of verbal communication, it can be observed that speaking a single sentence involves a highly complex decision making process. From the choice of selecting appropriate intonation of a syllable to the choice of the word, from the arrangement of the words in an orderly sequence to the adjustment of contour and timber, there are multiple decisions that the mind takes in a split second and it all happens with conscious awareness of the processes involved. If it is so then before arriving at a rational conclusion the mind contributes to a huge number of micro-rational steps that lead to the macro-rational finding. This process is certainly governed by a non-verbal capability of rationality. Contemporary science refers to this capability as non-verbal reasoning. Verbal reasoning, which in common parlance, known as rationality, is in fact just the tip of an iceberg. Same is true as far as aesthetic judgment is involved; the micro-rational processes responsible for coming up with rational ideas and theories also play a key role in determining the decisions.

The difference in products of research and drawing is due to the material used, visual appearance and set of skills involved. In research, the arguments are carefully spelled out, that takes into consideration on at least three accounts (i) the end product, (ii) the information available on the subject and (iii) an adjustment measure. The end product is what the researcher imagines to be the result of his intellectual labor. In the case of research, it can be a new interpretation or analysis which is closer to the truth of the matter involved. In terms of second consideration, a serious researcher goes through the available literature on the subject matter to avoid 'reinventing the wheel' or analyze observations in the light of established facts before putting them to writing. The third consideration is adjustment measure, which, in case of intellectual labor, is reason. The quality of rationality determines the originality and truth of the argument. The third consideration is interesting. David Bohm, a well-known physicist once said that amongst the contemporaries of Albert Einstein, there were various scientists who were better than Einstein in mathematical skill and knowledge but what Einstein had, was an eye for originality. ${ }^{7}$ Einstein himself was of the view that to understand the deeper laws of nature one needs a certain kind of aesthetic sensibility. It is therefore the third consideration or the quality of rationality that determines the fate of the research. Now rationality, in its most generalized sense, involves seeing things in the light of an underlying unity of order. This inevitably involves aesthetic sensibility for it is also something that has to do something with order and arrangement. Both rationality and aesthetic sensibility are

\footnotetext{
${ }^{6}$ Damasio, Antonio. Looking for Spinoza. (Oxford: Oxford University Press, 2005), p.38

${ }^{7}$ Bohm, David. On Creativity. (London: Routledge, 2004), p.4
} 
adjustment measures that a researcher and artist use in a creative act. Similarly, like a researcher, an artist uses observations as data and keeps the end product in mind.

Rationality, in modern academic discourse, has been classified in various ways. However, to confine the present discussion two classifications, epistemic rationality and strategic rationality are being discussed. These two modes of rationality are most clearly comparable to the kinds of rationality used by an artist. Epistemic rationality is "where the question is in the matter of fitting representation with the world. The goal is to reach the truth and avoid falsity. Epistemic rationality is the concern of logic and epistemology. Second form is strategic rationality where the concern is the fitting of the world and the goals set by the agent's desires and choices'. ${ }^{\mathbf{8}}$ It is suggested that these two kinds of rationality are largely governed by non-verbal reasoning and are comparable to the act of coming up with a product in a creative process of art. The true representation of what an artist observes involves epistemic rationality but since he or she does not use words and sentences to convey the representation, therefore, one does not see the representation of epistemic rationality. In fact, a true visual representation of a feeling has everything to do with epistemic rationality but in the non-verbal domain. The 'aesthetic sensibility' as a substitute adjustment measure to 'reason' continually makes adjustments. In the case of drawing, the movements of hand, the selection of the form of line corresponds to the feeling or theme that is to be expressed. The feelings or themes themselves are products of non-verbal reasoning and mostly conform to the second kind of rationality, i.e. strategic rationality. They mostly conform to the second kind for the simple reason that the artist is a subjectively motivated individual who does not limit himself to the objective truth of the outer world but is more inclined to see the aesthetic possibilities. The beauty and ugliness of the world affect him to the extent that he tends to see a modified world that is beautiful. It also happens that if his observation provides him with an ugly picture, he either shows that ugliness in its true measure, which involves epistemic rationality or keeps on representing brighter side through strategic rationality. The kinds of rationality are non-verbally deployed by the artist in a sense that they can be explained as two essential behaviors of aesthetic sensibility.

So, it can be suggested that the aesthetic sensibility of an artist in a creative act manifest two kinds of rational behaviors, i.e. epistemic and strategic. This holds true for a single creative event, that is, in production of a piece of art. Moreover, it also holds true if the evolution of representations in an artist's creative journey is analyzed. It is found that there is a correlation between his changing understandings of the world corresponding to a change in aesthetic preferences. To see this in practice, the case study of figurative treatment are being focused, wherein the aesthetic sensibility can be seen as performing the above mentioned rational behaviors both in terms of a single act of creation and as an evolving creative process. We will consider Sadequain's drawings in this regard since drawings are simpler than paintings and Sadequain has been an artist who portrayed his social and personal circumstances using both kinds of rationality.

\footnotetext{
${ }^{8}$ Markič, Olga. "Rationality and Emotions in Decision Making." Interdisciplinary Description of Complex
} Systems, (2009), pp.54-64. 


\section{Figurative Treatment in Sadequain's Drawings}

Considering the representation of human form in Sadequain's body of work, one realizes that there is a stylistic development taking place. He started with a realistic depiction of human form and moved to his famous cacti style in which human form was intermixed with cacti. Then we find a brief period of cubism leading to yet another kind of representation in which human form is portrayed deformed and distorted. Viewed from outside, these changes can be explained as four phases of stylistic development but if we observe from a psychological perspective, it can be suggested that the variation in style is correlated to a change in emotional and aesthetic judgments, which evolved vis-à-vis the intellectual position of the artist and his changing circumstances. According to the theoretical framework discussed in the above section, Sadequain's drawings are manifestations of epistemic and strategic rationality operating non-verbally. They were expressions of his feelings, which were, in turn outcomes of the way he thought and felt about his internal and external life. Sadequain's drawings therefore represent two layers of non-verbal reasoning. One belongs to the domain of 'perception'; where feelings come into being as emotional judgments and the other belong to 'execution'; where feelings are translated into a visual form guided by his aesthetic sensibility. It is the second layer that concerns the process of art that coincides with research.

The four phases in Sadequain's drawings namely (i) realistic, (ii) cacti, (iii) cubist, and (iv) exaggerated correspond to four phases of a general emotional state, which must have been resulted by a shift in Sadequain's understanding of the human being in a social setting. What we will be focusing here is a correspondence between the contents of the emotional state and the visual form. This correspondence will illustrate the hypothesis that the visual form involves adjustment measures in the spirit of research. The aesthetic sensibility of the artist silently performs the function of non-verbal reasoning. It is interesting to note that Sadequain himself explained the movement of his fingers being governed by something which he could not consciously explain. He said:

I did not have to move them [fingers], they moved, and moved the way they wanted to move; not as I wished them to move. For a while, I resisted the fingers; and forced them to move, as I wanted. It was strain. What was worse, the moment the controls were relaxed the fingers ran away. Thrice in a week, the fingers thus betrayed me; and upset my applecart. And when it came to starting anew fourth time, I gave up. After all, for all these years, the fingers had obeyed me; and so for a change I let the fingers be, and let them do their own biddings. ${ }^{9}$

The movement of fingers was certainly not a function, governed by fingers but more decisively by non-verbal reasoning.

Amongst the above mentioned four phases, the realistic and cubist phases were not explored at length by Sadequain; his focus was transitory as compared to the second and fourth phase. The realistic depiction can be explained by epistemic behavior of aesthetic sensibility since realistic depictions mean portrayals with maximum fidelity. For

${ }^{9}$ Shamim, Ahmed. "Treasures of Time." The Holy Sinner. (Karachi: Mohata Palace Museum, 2003), p.92 
example, an understanding of anatomy is necessary for realistic drawing regardless of drawing a nude or a figure with drapery. Figure drawings by Sadequain exhibit his knowledge of the proportions and sizes of the limbs and bones. In his work of 1950s, one can observe a remarkable perfection in realistic figure drawing; his competence cannot be denied in works such as Portrait of a Young Lady (Figure 1). It can be observed that he had a careful study of postures in relation to the positioning of the bones underneath the flesh. In the Observer Thinks (Figure 2), the form is exaggerated but still it involves a logical positioning of the bones since the figure is balanced on the right side of the body the emphasis has been added using the left side as well. The anatomy of the foot is again a very careful study; Sadequain kept track of the deformity in shape due to weight.

Consider the self-portraits by Sadequain. The accuracy of drawing is most noticeable, i.e. Self Portrait (Figure 3). It seems that Sadequain had a greater sensitivity for drawing the head. This would have required a detailed study of the skull. Sadequain would have studied the proportion of upper some and then the lower part of the skull which is smaller. The cheekbones and the circular outline of the eye socket have been drawn with spellbinding accuracy. It would have been a scientific bend of the artist that made him study and express the head's anatomy in exemplary detail. This fact becomes more obvious by looking at the various angles of head he has explored in his work. If the head is tilted, each and every part is aligned accordingly. One may assume that this scientific bend was due to epistemic rationality possessed by the artist.

The cubist phase expresses a mixture of epistemic and strategic rationality. It expresses strategic rationality as the world is fitting in to the desire of the artist, which is to show human form in a geometrical flow like Sitting Female (Figure 4). The epistemic rationality is involved since geometry deals with shapes and proportion. The mathematical nature of the style requires an epistemic attitude of aesthetic sensibility which shows fidelity towards proportion at one hand and then careful observation of how lines are perceived by the viewer in terms of direction and movement. In the phase inspired by cubism, Sadequain used directional lines as suggestive of movement. The use of these lines was correlated to certain feelings. For instance a horizontal layering often suggested a relaxed calmness in his drawings, whereas vertical lines would signify authority, strength, stiffness, striving etc. A diagonal or undulating line brought an element of action and activity to his drawings, i.e. The Frame of Figure (Figure 5). These drawings clearly express the viewpoint of a cubist, who is carefully handling the space.

Another correlation between elements of emotional state and visual form can be observed by analyzing the movement achieved through the use of line. Since emotions also involve a dynamic in addition to quality, Sadequain dealt with this aspect again with the use of 'flow of line'. In Under the Shower, Sadequain successfully controls the eye of the viewer through the use of directional lines as the eye travels from the lower part of the figure to the upper and then comes to a standstill on the head. The nature of directional lines as explored by Anwar refers to the usage of minimal number of lines, which are curved highlighting the course of droplets. The emphasis is on the hair which wraps the 
head pointing towards earring. ${ }^{10}$ Sadequain's signature lines are much closer to Picasso and Matisse. For instance, Couple Embrace by Picasso and Drawing of a Lady from Back by Matisse exhibit a similar quality of execution wherein flowing lines are noticeable. Barrington Barber explains the quality of Matisse's drawings in these words, "His [Matisse] drawings are marvelously understated yet graphic thanks to the fluidity of line. Awkwardness is evident in some of them, but ... they express exactly what he wanted. There are no extraneous marks to diffuse the image and confuse the eye". ${ }^{11}$ Sadequain expressed the dynamic in another way. He captured the images in action with fast speed drawings using simple lines and hatching, sometime with smudged tones. Ajaz Anwar writes for this crisscrossed moving figure entitled The Marathon (Figure 6), "With the impression of having run endlessly, the headless figure seems to be heading nowhere". ${ }^{12}$ The dim kaput strewn lines indicate the distance he has covered to show the complete movement. It is interesting to note that Sadequain, in terms of his epistemic and strategic rationality stands with Picasso and Matisse who expressed form through the use of directional lines and movement through fluidity of line.

The Cacti phase is a clear departure from known traditions. It was Sadequain's most original contribution to genre of drawing. This phase accounts for the brilliant illustration of strategic rationality. As defined earlier, strategic rationality refers to the ability wherein the world is modified to fit in the scheme desired or envisioned by the researcher. One can see that Sadequain perceived the social life of a human being as going through a continuous struggle against odd circumstances. He drew this inspiration while his stay at Gadani and by a detailed observation of Cacti. The visual form that he developed in the light of inspiration shows his strategic rationality to a level that one finds it difficult to distinguish that whether a cactus is transformed into human being or human being is transformed into cactus. The transformation was such that Hassan commented, 'The metamorphosis of the cacti into intriguing arrangements of monumental human forms is such that at times it becomes hard to distinguish between cactus and man'. ${ }^{13}$ His drawing, Transforming Cactus (Figure 7) represents a remarkable display of strategic rationality. To achieve the resemblance, he drew the fingers extraordinary long with various twigs resembling cactus with many nodes going upward. The emotional content for which he developed this cacti style are not a self-image. Sadequain himself was physically frail but the figures he drew were powerful and strong like cactus. The show resilience and power for struggle since they are born in a rocky landscape. The frailty is gone along with the tenderness. Their identity is full of the capability to defy whatever comes in the way. According to Safdar Mir, they symbolize Pakistani people who are full of strength and power with their strong survival instinct in harsh social reality. He further says that these symbols can be extended to all humanity

\footnotetext{
${ }^{10}$ Anwar, Dr. Ajaz. Sadequain Artiste de Pakistan "The Missing Link". (Lahore: A Heritage of Pakistan Publication, 2004), p.49

${ }^{11}$ Barber, Barrington. Advanced Drawing Skills. (London: Arcturus Publishing Limited, 2004), p.40.

${ }^{12}$ Anwar, Dr. Ajaz. Sadequain Artiste de Pakistan "The Missing Link". (Lahore: A Heritage of Pakistan Publication, 2004), p.96

${ }^{13}$ Hassan, Ijaz Ul. Painting in Pakistan. Revised $2^{\text {nd }}$ ed. (Lahore: Ferozsons \{Pvt.\} Ltd, 1996), p.81
} 
since the fight for life is everywhere. The struggle is manifest wherever death and destruction are confronted. ${ }^{14}$

However, Sadequain also had a positive vision regarding cactus. In an interview, he told Rehana Hakim about his use of cactus, "To me [cactus] symbolizes the triumph of life over the environment.... I used to identify it with my own self. Then I started identifying it with the whole community'. ${ }^{15}$ According to Marcella Sirhandi, 'The burning red sun and dry, sterile environment are mocked by the triumphant cactus people. They thrive, proliferate and grow to army strength. They represent the forces of reason countering those of barbarians'. ${ }^{16}$ Ijaz ul Hassan described the dual character of cactus symbolism as man's struggle against natural hazards (like the cacti's against the harsh elements of the desert) and his ultimate victory over them, and additionally, its ugly, hairy, and thorny form is exaggerated to represent man's moral decadence. ${ }^{17}$ All these artists and scholars can clearly sense the symbolism as a profound innovation by the great master. It was certainly an expression which was much thought-out and was studied in depth and implications. The significance of the symbol came from Sadequain's own experience.

The fourth phase became a hallmark of Sadequain's style. He distorted the forms and exaggerated the proportion. Distortion and exaggeration are devices that he used to portray man's ugliness, which was a state coming out of man's resistance to evolve emotionally and intellectually. This had much to do with Sadequain's intellectual position regarding the ugliness of stagnant existence. It is interesting that in order to express in exactness he rejected the stylistic conventions that were a product of Western societies, he distorted forms and created tortured figures. Deformation and distortion added pain and agony to the picture. ${ }^{18}$ The exaggerated form does not represent a physical distortion of human being but a spiritual state of helplessness, material opportunism, and moral decline.

In Sadequain's own poetry, one can find windows to what he felt at the spiritual plane. For instance the following Rubayi:

How long shall I hold a mirror to my obsession?

How long shall I suffer in this world;

How long shall I suffer in the grief of life;

How long shall I suffer the burden of creativity? ${ }^{19}$

\footnotetext{
${ }^{14}$ Mir, Safdar. "A Thinker Through Images." The Holy Sinner. (Karachi: Mohatta Palace Museum, 2003), 80-81

${ }^{15}$ Hakim, Rehana. "Sadequain is a showman because he has something to show." The Herald, July 1985: 110117

${ }^{16}$ Sirhandi, Marcella Nesom. Contemporary Painting in Pakistan. (Lahore: Ferozsons Pvt. Ltd, 1996), 58.

${ }^{17}$ Hassan, Ijaz Ul. Painting in Pakistan. Revised $2^{\text {nd }}$ ed. (Lahore: Ferozsons \{Pvt.\} Ltd., 1996), 81.

${ }^{18}$ Manzoor, Shahida. "Sadequain: Conception and Misconceptions." (n.d.).

${ }^{19}$ Anwar, Dr. Ajaz. Sadequain Artiste de Pakistan "The Missing Link". (Lahore: A Heritage of Pakistan

Publication, 2004), p.56
} 
This phase is a precise expression of strategic rationality. Sadequain had to express both the intensity and quality of spiritual deformation; therefore, he chose exaggeration and deformation as stylistic conventions.

\section{Limitation of the Study}

The study involves a comparison between the verbal and non-verbal processes of the human mind in relation to creative problem solving. The study does not include empirical investigations to the collected data and analysis for the purposes of study. It only corroborates evidence that is available in literature and that too is not exhaustive. However, it does initiate a debate in the area of study. It has yet to be seen that what has been counter evidence in this scenario. It might be possible that more mental processes could be seen as playing a decisive role in the research that has been associated to drawing process. The reason is the artistic intent involved informed by aesthetic preferences difficult to be quantified. The purpose of the present study was to highlight its role in creativity. Empirical investigations into questions such as how emotions play a role in decision making and how the underlying processes of verbal reasoning and creativity in drawing are linked due to similar mental mechanisms involved will bear more fruit and may contribute to a clearer understanding.

\section{Conclusion}

The mental processes that determine verbal and non-verbal reasoning are similar on many grounds. It has been shown in various studies that emotions play a vital role in decision making, and, emotions are results of non-verbal calculations performed by human mind at a non-conscious plane. In light of this the problem solving capability of the mind is general, however, the development of skill in a particular medium may contribute to an enhanced application of the capability. Philosophers, scientists and artists all use the problem solving capability but in different mediums. The research process involves application of mental processes in creative ways and same goes for the artist who produces a drawing. The languages might differ but the mental actions remain similar.

Sadequain's treatment of human in four distinct ways explains that his understanding about human condition and its presentation on canvas evolved throughout his life. Although, there has been a serious intellectual labor that goes into his artistic genius but more significant was his sensitivity to pain. The feelings that he expressed were a product of non-verbal reasoning and in the same spirit their translation into visual form was also a product of his sensitivity to the medium. He used both epistemic and strategic modes of rationality to express his feelings and embodied the spirit of research in art process. In this paper, a brief review of Sadequain's treatment of human figure and the use of line is presented, but on a closer observation one finds that from each element to each principle of art that he used there were reasons that only one's heart knows. If the essence of what he achieved through his various drawing could be translated in a verbal discourse, it would seem as thoroughly spelled out a rational argument. 


\section{Figures}
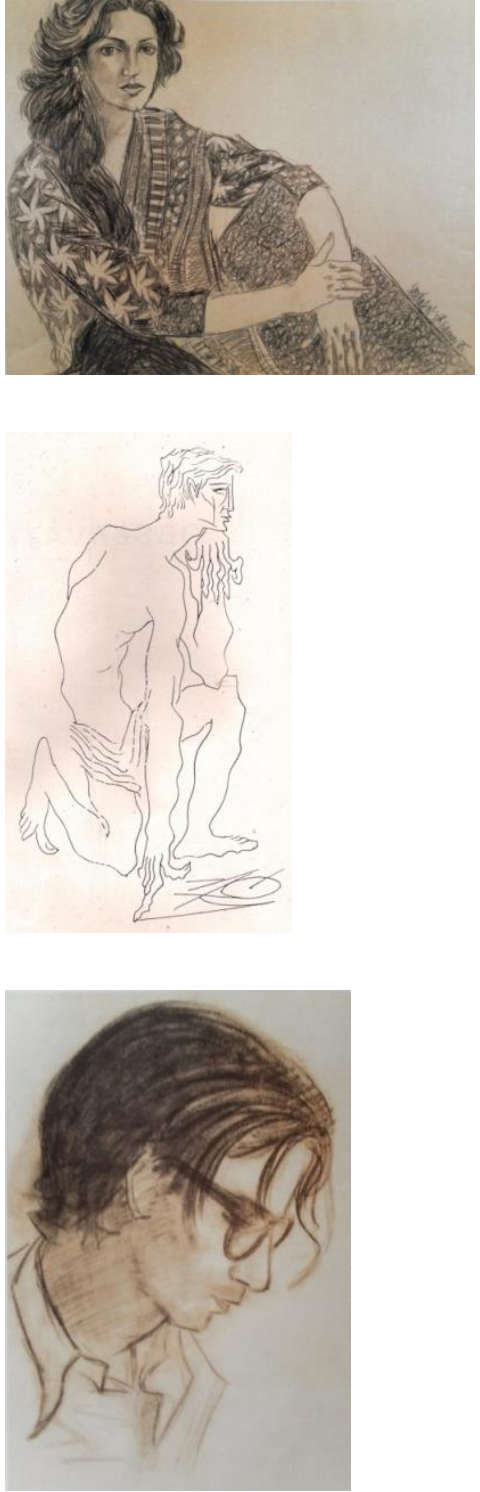

Figure 1: Sadequain,

The Dark Lady at Ease 1

1985

Pen and Ink on Paper

Size $65 \mathrm{~cm} \mathrm{x} 75 \mathrm{~cm}$

(Museum 2003, 433)

Figure 2: Sadequain, The Observer Thinks 1970

Pen and Ink on Paper (Sadequain 1984, 78)

Figure 3: Sadequain, Self Portrait 1960

Size $48 \mathrm{~cm} \times 36 \mathrm{~cm}$ Oil Pastel on Paper (Museum 2003, 180) 

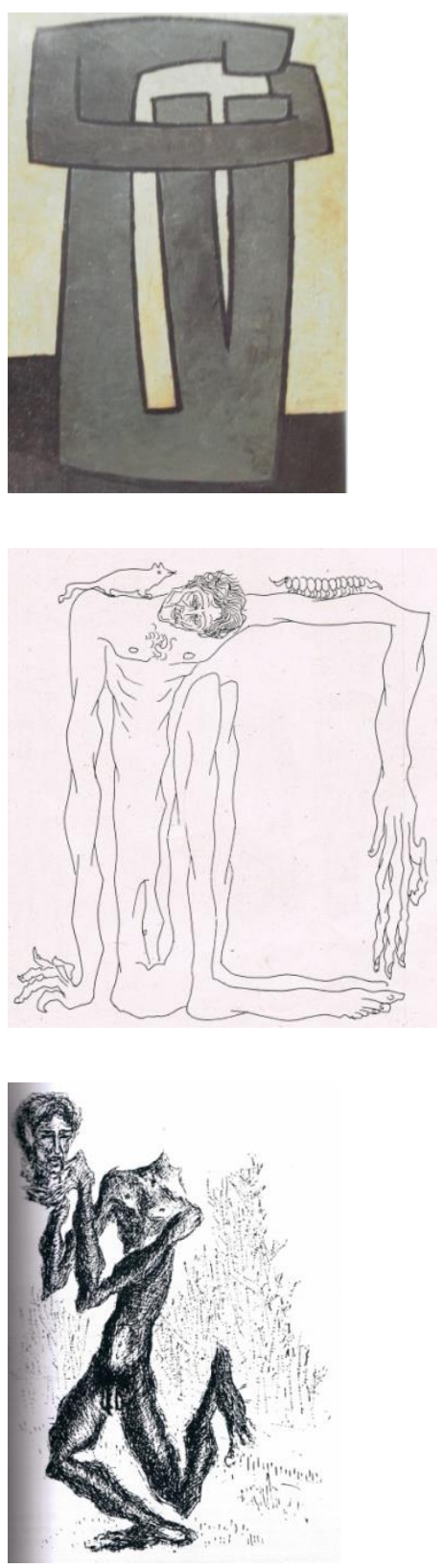

Figure 4: Sadequain, Sitting Female

Cubist Style

1961

(Museum 2003, 178)

Figure 5: Sadequain, Frame of Figure 1970 Pen and Ink on Paper (Sadequain 1984, 61)

Figure 6: Sadequain, The Marathon

Pen and Ink on Paper

Size 22x30 Inches

(Anwar 2004. 97) 


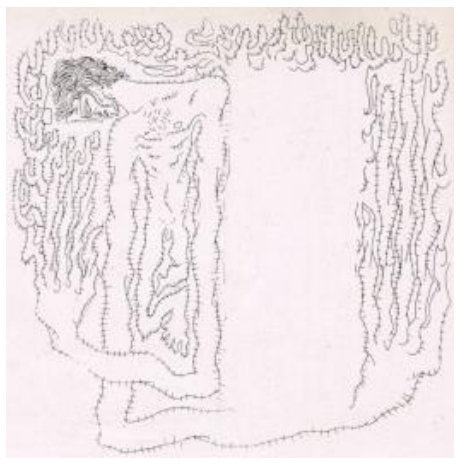

Figure 7: Sadequain,

The Transforming Cactus 1970 Pen and Ink on Paper (Sadequain 1984, 62)

\section{Glossary}

Aesthetic sensibility: The ability to perceive aesthetically the nature of art, beauty and taste with the creation and appreciation or sensation of beauty.

Drawing conventions: Ways in which drawing is usually done or an agreement between states covering particular matters of drawing, especially less formal than harmony.

Emotional processes: Presentation or activation of fear incompatible relevant information.

Evolutionary: The process of development or the theory, that something or a statement has grown and developed from past things or some previous statements.

Hierarchy: A system in which members of a group or society are ranked one above another according to relative status or authority.

Metaphor: a figure of speech in which a word or phrase is applied to an object or action to which it is not literally applicable.

Neurobiologists: A branch of science that deals with the anatomy, physiology, and pathology of the nervous system.

Non-Verbal Reasoning: That involves the ability to understand and analyze visual information and solve problems using visual reasoning without involving or using words or speech.

Rationality: The quality or state of being reasonable based on facts or reason.

Subconscious: The part of consciousness that is not presently in focal awareness or it concerns the part of the mind which is not fully aware but that influences one's actions and feelings. 


\section{Bibliography}

Anwar, Dr. Ajaz. Sadequain Artiste de Pakistan "The Missing Link". Lahore: A Heritage of Pakistan Publication, 2004.

Barber, Barrington. Advanced Drawing Skills. London: Arcturus Publishing Limited, 2004.

Bohm, David. On Creativity. London: Routledge, 2004.

Damasio, Antonio. Looking for Spinoza. Oxford: Oxford University Press, 2005.

Hakim, Rehana. "Sadequain is a showman because he has something to show." The Herald, July 1985: 110-117.

Hassan, Ijaz Ul. Painting in Pakistan. Revised 2nd Edition. Lahore: Ferozsons (Pvt.) Ltd., 1996.

Lewicki, Paul. Conclusions of the Research on Nonconscious Information Processing. July 3, 2013.

Lipton, Bruce. The Power of the Mind. January 1, 2008.

http://www.newdawnmagazine.com/articles/the-power-of-the-mind (accessed October $15,2014)$.

Manzoor, Shahida. "Sadequain: Conception and Misconceptions." (n.d.).

Markič, Olga. "Rationality and Emotions in Decision Making." Interdisciplinary

Description of Complex Systems, 2009: 54-64.

Mir, Safdar. "A Thinker Through Images." The Holy Sinner. Karachi: Mohatta Palace Museum, 2003. 80-81.

Museum, Mohata Palace. The Holly Sinner. Karachi: Mohata Palace Museum, 2003.

Shamim, Ahmed. "Treasures of Time." The Holy Sinner. Karachi: Mohata Palace Museum, 2003.

Sadequain. Sadequain Drawings August 1970. Karachi: Noor Company, 1984.

Sirhandi, Marcella Nesom. Contemporary Painting in Pakistan. Lahore: Ferozsons Pvt. Ltd, 1996.

Tooby, Leda Cosmides \& John. Evolutionary Psychology and the Emotions. January 13, 2000. http://www.cep.ucsb.edu/primer.html (accessed October 8, 2014). 